\title{
Phylogenetic Placement and Taxonomic Reinvestigation of Endemic and Endangered Plant Species: Silene Leucophylla Boiss. and Silene schimperiana Boiss. (Caryophyllaceae)
}

\author{
Ahmed EL-Banhawy ${ }^{1, *}$, Faten Y Ellmouni ${ }^{2, *}$, Iman H Nour ${ }^{3}$, Ahmed Faried ${ }^{4}$, Asmaa O. Olwey ${ }^{5}$ \\ and Ahmed ElKordy ${ }^{6}$ \\ 1 Botany Department, Faculty of Science, Suez Canal University, Ismailia, Egypt \\ 2 Botany Department, Faculty of Science, Fayoum University, Fayoum, Egypt \\ 3 Botany and Microbiology Department, Faculty of Science, Alexandria University, Alexandria, Egypt. \\ Iman-hassan@hotmail.com \\ 4 Botany and Microbiology Department, Faculty of Science, Assiut University, Assiut, Egypt; \\ ahmedfaried55@aun.edu.eg \\ 5 Botany and Microbiology Department, Faculty of Science, Assiut University, Assiut, Egypt; \\ asmaa_olwey@science.aun.edu.eg \\ 6 Botany and Microbiology Department, Faculty of Science, Sohag University, Sohag, Egypt; \\ aelkordy@science.sohag.edu.eg \\ * Correspondence: ahmedbanhawy@science.suez.edu.eg (A.E.-B.); fyl00@fayoum.edu.eg (F.Y.E.)
}

Published: 30 November 2020

\begin{abstract}
The two endemic plant species Silene leucophylla and Silene schimperiana (Caryophyllaceae) are native to the Sinai Peninsula which considered as one of the floristically richest phytogeographical hot spot regions of the Mediterranean basin. The acquaintance of Sinai's biodiversity is crucial for conservation and sustainable development. Endemic plant species of Sinai are vulnerable to anthropogenic threats due to their relatively low population size. The current study reinvestigated the taxonomic status of two medicinally important and Endangered species. The integrated approach of macro and micro-morphological traits using a scanning electron microscope (SEM) as well as phylogenetic analysis were conducted. Phylogenetic reconstruction using Bayesian Inference based on DNA sequences of nuclear (ITS) and chloroplast ( $r b c l$ and matk) markers retrieved the species phylogeny successfully. Silene leucphylla and Silene schimperiana was placed phylogenetically within the whole genus. The sectional classification of the two species was confirmed. Silene leucophylla was placed in section Siphonomorpha while Silene schimperiana allied to section Sclerocalycinae. The current study reassured that the integration between the various morphological and molecular approaches is substantial to identify, determine the taxonomic status, and reveal the phylogenetic position of those two endangered plant taxa.
\end{abstract}

Keywords: endangered; endemic; Silene; SEM; stomata; molecular systematic; phylogenetic analysis; nrDNA ITS; cpDNA matk

\section{Introduction}

Anthropogenic risk and environmental transformations are ordinarily believed to be a higher extinction threat for endemic plant species because they are extra vulnerable [1]. Nowadays the community is more conscious of the worth of endemic plant species, and their distinct genetic composition, hence they have a high-priority to conserve them [1,2]. Egypt is situated in the southeast of the Mediterranean coast which almost contains $7 \%$ of the plants all around the world [1,2]. Abdelaal et al. [3] recorded 48 endemic taxa in Egypt.

Silene L. (tribe Sileneae) is considering one of the largest genera in Caryophyllaceae with about 850 species distributed in the Northern Hemisphere, temperate regions of the Mediterranean zone in 
addition to central and western Asia [4,5]. Jafari et al. [4] divided Silene into of three subgenera (Lychnis, Behenantha, and Silene) as well as 34 sections, using morphological and phylogenetic analysis. Twenty nine Silene taxa were recognized in Egypt three of them are endemic S. leucophylla Boiss., S. schimperiana Boiss. and S. oreosinaica [6-10]. S. leucophylla and S. schimperiana have been allied to subg. Silene, below sections Siphonomorpha and Sclerocalycinae respectively [10-12]. Macro and micromorphological characteristics of leaf epidermal cells have important taxonomical features and have a vital role in the discrimination between taxa within family Caryophyllaceae and for discrimination of Silene taxa at the specific level [13-16].

The phylogenetic analysis is essential for empathetic structural and functional characteristics of biodiversity in an evolutionary background [17]. Therefore, utilization of the phylogenetic data is important for ecological, taxonomical, and evolution studies $[18,19]$. Phylogenetic relationships of large genus like Silene is always considered challenging. A new taxonomic underpinning for the infrageneric classification of Silene species based on nrDNA ITS and cpDNA rps16 sequences has been conducted by Jafai et al. [4]. In the current study morphological and molecular phylogenetic data of S. leucophylla and S. scimperiana species were merged. The current study aims to contribute the species designation, identification, and revealing the phylogenetic position of those endemic species within the whole genus.

\section{Experiments}

\subsection{Plant Materials}

For morphological and anatomical analyses, herbarium specimens of S. leucophylla and S. schimperiana were obtained from ASTU herbarium. For molecular analysis, fresh leaf materiales were collected from different populations across its geographic distribution at Saint Katherine, South Sinai, Egypt.

\subsection{Morphological and Anatomical Analyses}

The stem as well as the leaf abaxial (AB) and adaxial (AD) surfaces were mounted onto stubs with double-sided adhesive tape, coated for $5 \mathrm{~min}$ with gold in a polaron JFC-1100E coating unit, then were examined and photographed with JEOL JSM-IT200 scanning electron microscope unit at Faculty of Science, Alexandria University, Alexandria, Egypt. The quantitative characters were measured by image analysis software [20] and the terminology of Barthlott et al. [21] were followed.

\subsection{Statistical Analysis}

All quantitative data applied by using the R- software with the required packages installed [22]. Boxplots were created using the "ggplot2" library [23]. Analysis of variance (ANOVA) was performed using (aov) function. Which it followed by Post Hoc Tukey Honestly Significant Difference (HSD). The "pheatmap" and "ggplot2" packages [23,24], were used to visualize the similarity and dissimilarity within and among two species. The "corrplot2" package was used to visualize the correlation output by drawing the correlogram [25].

\subsection{Phylogenetic Analysis}

DNA Extraction, PCR Amplification, Sequencing, and Phylogenetic Analysis

Fresh leaf materials used for molecular analyses were collected and preserved in silica gel. DNA was extracted using the Cetyltrimethylammonium bromide (CTAB) protocol with some modifications [26]. The PCR amplification performed in $15 \mu \mathrm{L}$ volume for ITS, and matK, containing $5 \mathrm{U} / \mu \mathrm{L}$ Taq DNA polymerase with $25 \mu \mathrm{M} \mathrm{MgCl} 2,10 \mu \mathrm{M}$ of dNTPs, $10 \mu \mathrm{M}$ of each primer. Amplifications were conducted using an Applied Biosystems ${ }^{\circledR}$-VeritiTM 96- well thermal cycler. PCR products were purified with ExoSAP-IT (USB Corporation, Cleveland, OH, USA). PCR products 
were sent to Macrogen Spain for direct sequencing in both directions with an ABI 3730XL Genetic Analyzer (Life Technologies Corporation).

These novel DNA sequences of S. leucophylla and S. scimperiana were deposited in the GenBank under the accessions ITS: (submitted to GB), and matK: (submitted to GB). The aligned DNA sequences for ITS and matKthe were used to construct two single markers and a combined dataset. The optimal nucleotide substitution model was estimated using MrModeltest [27], and executed in MrBayes blocks. A 50\% majority role consensus tree was constructed to get the posterior probabilities (PP). Posteriori probabilities, values $>0.5$ at a given branch were considered strong support for the existence of that branch [28].

\section{Results}

Stem and leaf qualitative and quantitative characteristics are summarized in (Appendixes A and B), respectively.

\subsection{Stem Micromorphology}

The stem surface was covered by unicellular non-glandular pustulate trichomes of size 45-98 $\times$ 13-23 $\mu \mathrm{m}$ in S. leucophylla Figure 1a,b, whereas S. schimperiana had glabrous surface Figure 2a. The stem epicuticular wax was film-like in S. leucophylla and irregular flat crystalloid platelets $(<1 \mu \mathrm{m}$ height) with sinuate margin in S. schimperiana. The type of stomatal complex was anomocytic in the investigated species.

Geom_boxplot and ANOVA of the quantitative data were applied for measured stomatal characteristics (length, width and area) and stomatal pore (length, width and area). Which shows and confirms the stomatal variations of the two species with anlysis osignificant $p$-value $\left(p=0.0054^{* *}, R\right.$ squared $=0.9487)$, that showed the higher median at stem stomata of S. leucophylla $\left(p=0.00713^{* *}\right)$ than S. schimperiana $\left(p=0.00563^{* *}\right)$ Figure 3a.
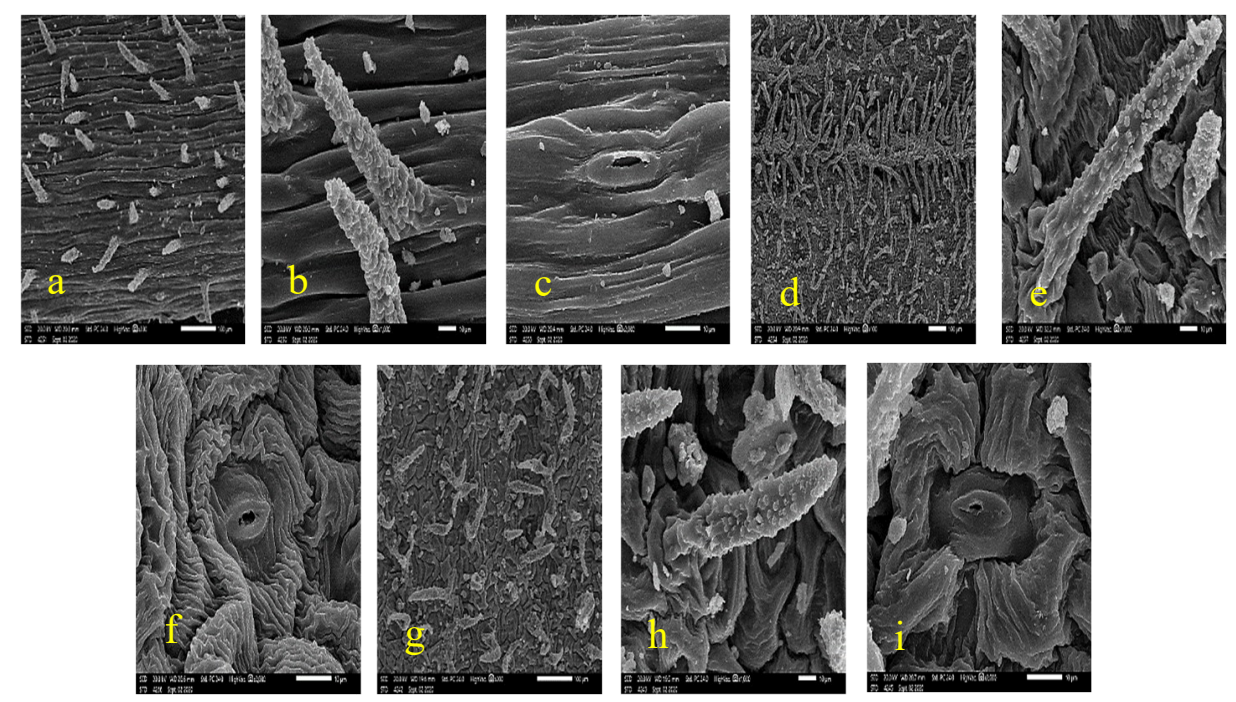

Figure 1. Scanning Electron Microscope (SEM) photo-micrographs of Silene leucophylla. (a-c) stem; (di) leaf. (a) surface, (b) trichome, (c) stomata, (d) abaxial surface, (e) abaxial trichome, (f) abaxial stomata, (g) adaxial surface, (h) adaxial trichome, (i) adaxial stomata.
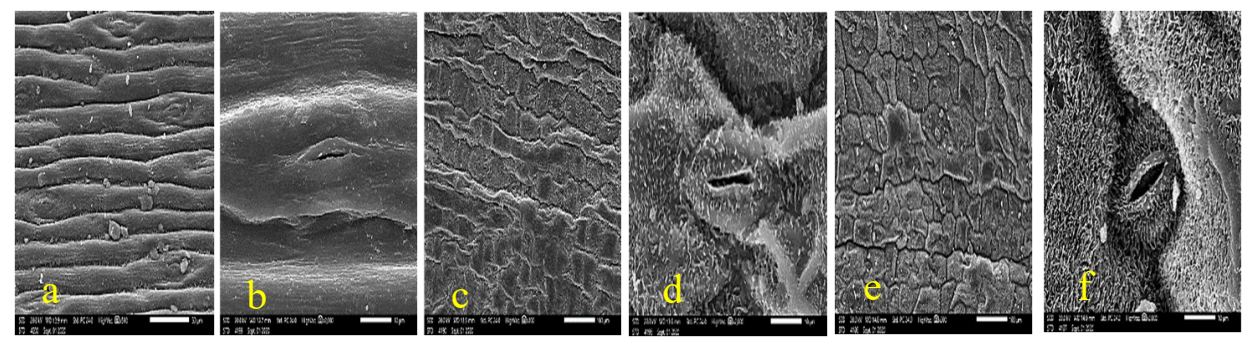
Figure 2. SEM micrographs of Silene schimperiana. (a,b) stem; (c-f) leaf. (a) surface, (b) stomata, (c) abaxial surface, (d) abaxial stomata, (e) adaxial surface, (f) adaxial stomata.

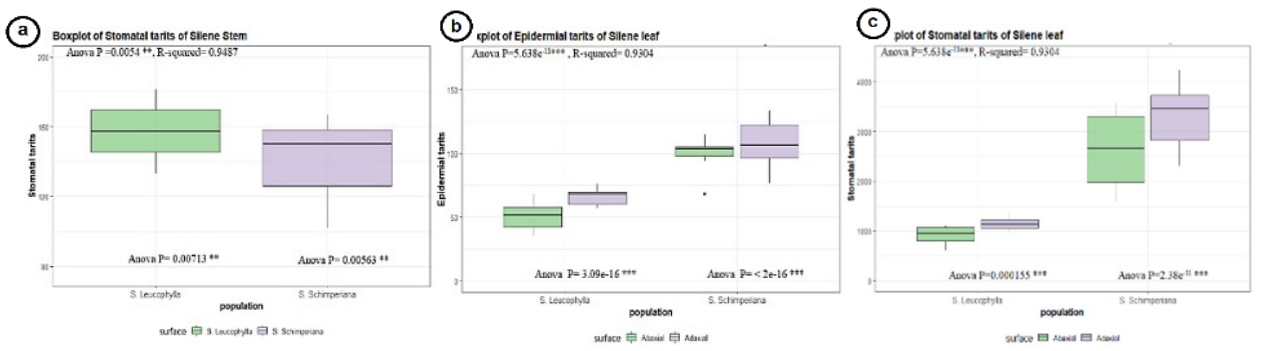

Figure 3. boxplots of the quantitative data for (a) stomatal characteristics at the Stem micromorphology with the stomatal (length, width and area) and stomatal pore (length, width and area) in endemic S. leucophylla and S. schimperiana. (b) epidermal cells size at the epidermal cells micromorphology (length and width) in endemic S. leucophylla and S. schimperiana. (c) of stomatal characteristics with the stomatal complex, subsidiary cells and stomatal pore (length, width and area) respectively in endemic $S$. leucophylla and S. schimperiana.

\subsection{Leaf Epidermal Cells}

The epidermal cell characteristics were separately described for abaxial (AB) and adaxial (AD) leaf surfaces. For the primary sculpture; leaf epidermal cells were parallel or irregularly arranged, and their shapes were oblong to bone-shape or tetra-, penta-, hexa- to polygonal. Significant variations were distinguished in size of epidermal cells with Anova $p<2.2 \times 10^{-16 * * *}, R$-squared $=$ 0.9708 , the smallest epidermal cell on both surfaces were observed in S. leucophylla; on AB surface $(23.51-42.76 \times 8.73-19.25 \mu \mathrm{m})$ Figure $1 \mathrm{~d}$ and on AD surface $(31.16-52.41 \times 12.79-26.97 \mu \mathrm{m})$ Figure $1 \mathrm{~g}$. While, the largest cell on both surfaces were observed in S. schimperiana on AB surface (30.52-61.40 $\times$ 37.85-63.79 $\mu \mathrm{m})$ Figure 1c and on AD surface $(35.48-91.04 \times 32.47-80.59 \mu \mathrm{m})$ Figure 1e. Which confirmed by grouped boxplot for abaxial and adaxial leaf Figure $3 b$.

The Anticlinal Walls (AW) were usually sunken, irregularly curved in S. leucophylla or straight in S. schimperiana. The relief of cell boundary was generally channeled, it is deeply ribbed on the AB surface and slightly ribbed on AD surface in S. leucophylla or smooth in S. schimperiana. For the secondary sculpture; fine relief of the cell wall was regular striate cuticular sculpture in S. leucophylla and smooth in S. schimperiana. For the tertiary sculpture; epicuticular secretions are similar to those found on the stem of both S. leucophylla and S. schimperiana.

\subsection{Stomatal Complex}

Leaves are amphistomatic in the two studied species. The raised diacytic type of stomata was observed in S. schimperiana, while S. leucophylla attained both sunken diacytic and tetracytic stoamta. The surface of the guard cells was either smooth in S. leucophylla or epicuticular crustose platelets in S. schimperiana. The smallest stomatal area was recorded in S. leucophylla on the AB surface (46.44$\left.74.64=61.42 \pm 8.56 \mu \mathrm{m}^{2}\right)$ Figure $1 \mathrm{f}$, and the largest area was recorded in S. schimperiana on the AD surface $\left(102.20-253.50=152.30 \pm 37.92 \mu \mathrm{m}^{2}\right)$ Figure 1i. Grouped boxplot for abaxial and adaxial leaf for variation at stomatal pore, stomatal complex and subsidiary cells of Length, width and area respectively with Anova $p=5.638 \times 10^{-11 * * *}, R$-squared $=0.9304$, that proofed the previous measuring reading Figure 3c. Moreover, the lowest Stomatal Index (SI\%) was recorded in S. leucophylla (11.76$12.12=11.94 \pm 0.25)$, and the highest SI $(12.90-20.69=15.10 \pm 3.76)$ was recorded on the AD surface in S. schimperiana.

Finally, pheatmap exhibited the variation between understudies' two taxa, where in the two $S$. leucophylla replicate measured reading grouped together at separate cluster which divergence from three S. schimperiana replicate revealed little divergence within it, whereby they collected from two 
places with little difference of altitude range Figure 4a. The Correlogram correlation analysis exposed significant relationship among numerous traits (Figure $4 b$ ).
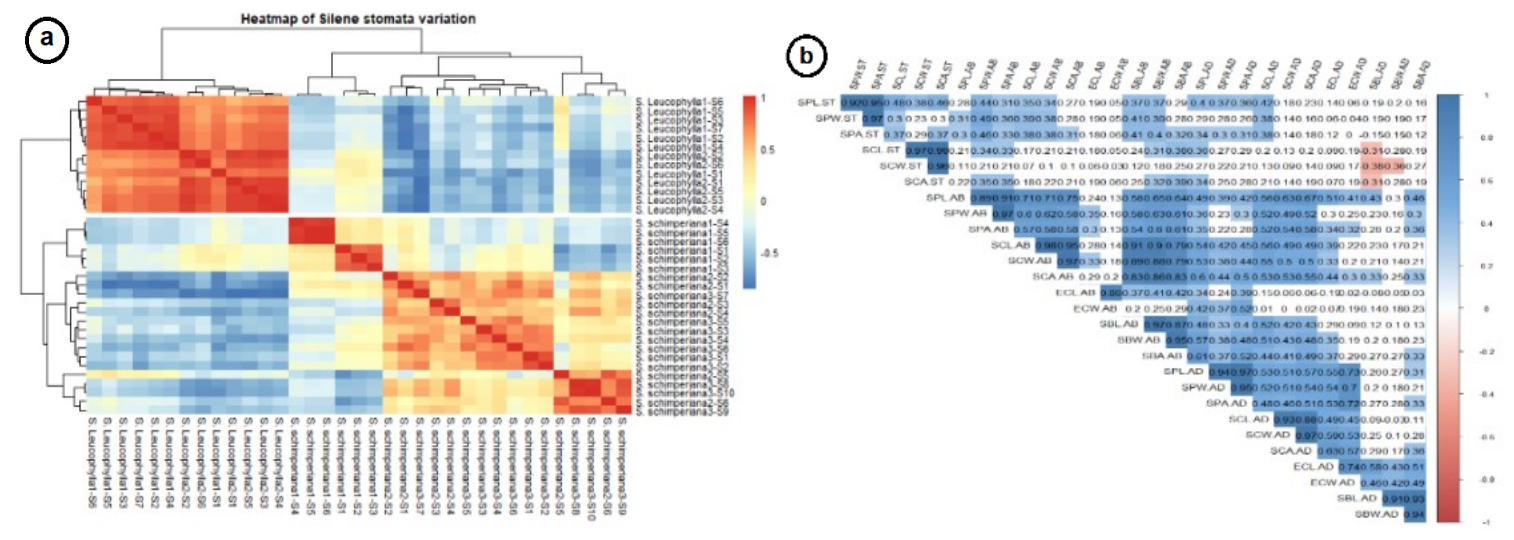

Figure 4. (a) pheatmap for based on the quantitative data of Stem and leaf micromorphology traits, to visualize the similarity and dissimilarity within and among S. leucophylla and S. schimperiana. (b) Correlogram for quantitative traits. Positive and negative correlations are displayed in blue and in red color, respectively. Correlation coefficients are proportional to color intensity, and the size of the circle is proportional to the correlation coefficients.

\subsection{Phylogeny}

In the combined nrDNA ITS and cpDNA matK circularized phylogenetic tree (Figure 5), Atocion lerchenfeldianum, Atocion rupestre, Viscaria alpina, Arenaria densissima, Arenaria polytrichoides, Arenaria smithiana and Bufonia multiceps composed the outgroup taxa. The in group consists of 33 Silene taxa. The three strong supported sections: "S. sect. Auctifolia (Posterior Propability PP = 100), S. sect. Auriculate ( $P P=100)$, and S. sect. Silene ( $P P=100)$ " were represented by S. cordifolia, S. schafta, and S. ciliata. Silene leucophylla was placed in Silene sect. Siphonomorpha s.l. $(P P=100)$, while S. schimperiana was placed in S. sect. Sclerocalycinae s.l. $(\mathrm{PP}=98)$. 


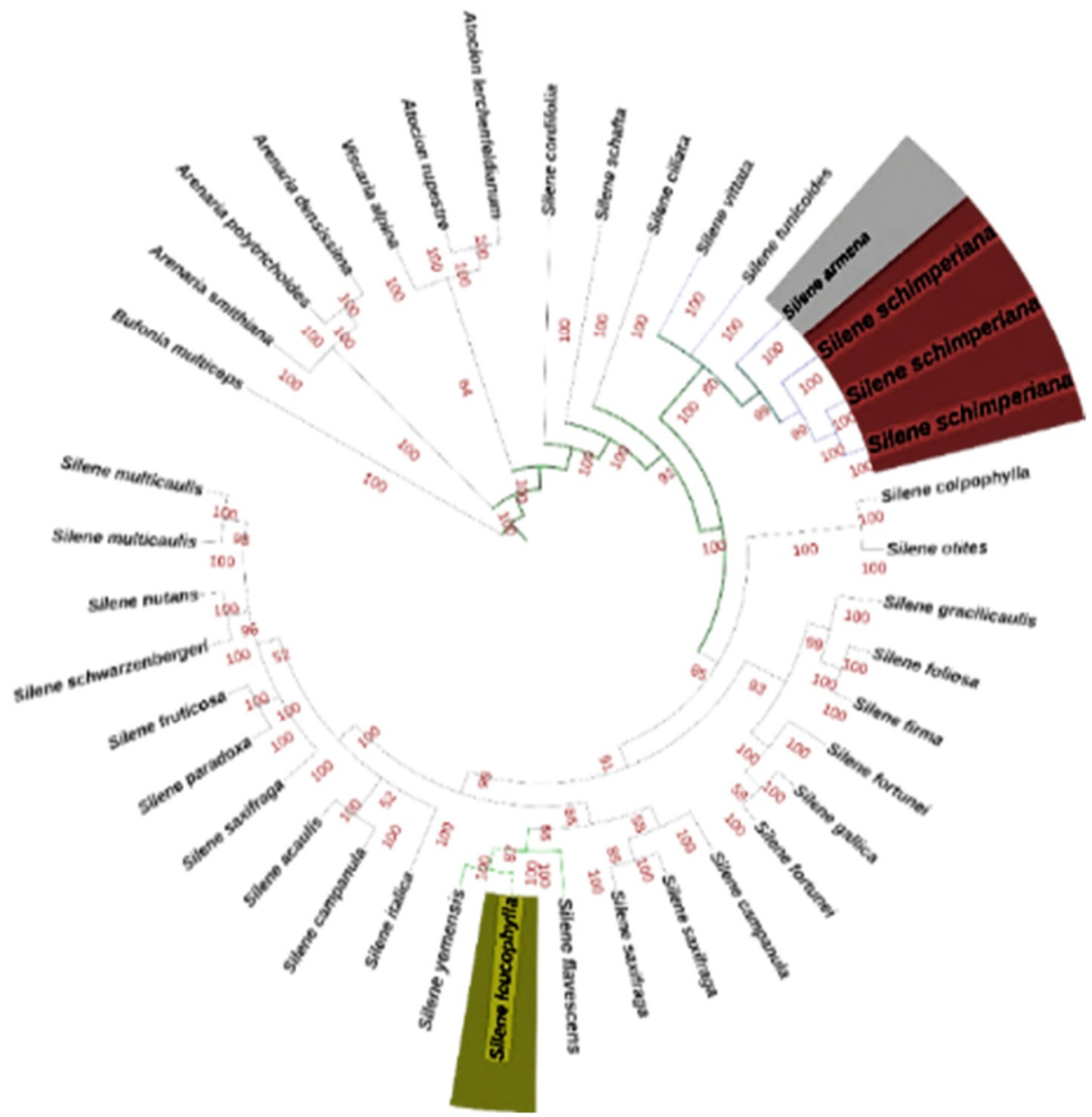

Figure 5. Bayesian phylogenetic tree based a combined DNA sequences of nrDNA ITS and cpDNA matK.

\section{Discussion}

The current study represented the first detailed leaf-surface morphology for the Egyptian endemic and near endemic taxa; S. leucophylla and S. schimperiana respectively. Stem and leaf of $S$. schimperiana are characterized by the presence of epicuticular secretions, in the form of irregular flat crystalloid crustose platelets. These waxes have a great systematic significance and ecological importance due to the interaction between plants and their environment [21]. Furthermore, both stem and leaf surfaces were covered by unicellular non-glandular pustulate trichomes in S. leucophylla, whereas S. schimperiana had a glabrous surface. Our results are compatible with Stace [29], who suggested that anticlinal wall patterns are corresponding to the habitat environment, where species growing in drier habitats attain straight to curved anticlinal walls.

The studied species are amphistomatic which is a characteristic feature for species occupying xerophytic habitats [30]. In the present study, stem stomatal complex area in S. leucophylla is 1.2 times larger than S. schimperiana. On the other hands, leaf measurements of stomatal complex and epidermal cells characteristics were always greater on the AD surface than on the AB surface for both species, as well as size of $S$. schimperiana cells is larger than S. leucophylla. It was notable that the former cell size of stomatal complex area is 2.6-2.9 times, stomatal pore area is 24.4-33.5 times and subsidiary cell area is 2.69-3.13 times larger than the latter. As mentioned by Rossatto and Kolb [31], species located in shady areas similar to the top of Saint Katherine Protectorate mountains had a low average Stomatal Index (SI\%). That is harmonious with our study as SI is $12.42 \%$ and $14.44 \%$ for S. leucophylla 
and S. schimperiana respectively. In contrary, high average of SI (up to $95.58 \%$ ) is noticed for species found in sunny areas [32].

Rohrbach [33] classified S. leucophylla and S. schimperiana at the same Sectio III. Botryosilene, but differ at Series 8. Nutantes, and 1. Sclerocalycinae, respectively. While Chowdhuri [10] and Hosny, et al. [12] placed it with sect. Siphonomorpha and sect. Sclerocalycinae(Subsection Chlorifoliae), respectively. The latest studies Oxelman et al. [11] deals with S. leucophylla as S.subsect. Brachypodae, allied to S. sect. Siphonomorpha. While S. schimperiana to S. subsect. Sclerocalycinae.

Our results phylogenetic studies a combined phylogenetic tree nrDNA ITS and cpDNA matK confirm that S. leucophylla related to sect. Siphonomorpha, due to its noticeable S. leucophylla shared S. yemensis in the same clade and in-group with $S$. flavescens, all over related to S. sect. Siphonomorpha. While $S$. schimperiana allied to $S$. subsect. Sclerocalycinae, which exhibited at a clade in a group with $S$. armena, and S. tunicoides wherein related to S. sect. Sclerocalycinae. That is in line with Jafari et al. [4] phylogenetic studied but he didn't examine the two endemic Egyptians under study.

\section{Conclusions}

In conclusion, Stem and leaf micromorphology (SEM) revealed the complete distinction between two taxa and discuss the variation between them that permitted the endemism of S. leucophylla and S. schimperiana. While the phylogenetic studies confirm the classification of them that to the relevant section that classified depended only on the morphological description.

Author Contributions: Conceptualization A.E.-B., A.A.F. and A.F.; methodology, A.E.-B. and D.A.U., software, A.E.-B. and D.A.U.; formal analysis, A.E.-B., D.A.U., and A.F.; data curation, A.E.-B., D.A.U., and M.M.; investigation, A.E.-B., F.Y.E., I.H.N. and A.F.; resources, A.E.-B., I.H.N. and A.O.O.; Formal analysis, A.E.-B., F.Y.E., A.O.O. and A.E.; Data curation, A.O.O. and A.E.; writing-original draft preparation, A.E.-B., F.Y.E., I.H.N., A.F., A.O. and A.E.; writing-review and editing, A.E.-B.., F.Y.E., I.H.N. and A.F; funding acquisition, A.E.-B., F.Y.E., I.H.N., A.F., A.O,O, and A.E.; All authors have read and agreed to the published version of the manuscript.

Conflicts of Interest: The authors declare no conflict of interest.

\section{References}

1. Coelho, N.; Gonçalves, S.; Romano, A. Endemic Plant Species Conservation: Biotechnological Approaches. Plants 2020, 9, 3, 345.

2. Foggi, B.; Viciani, D.; Baldini, R.M.; Carta, A.; Guidi, T. Conservation assessment of the endemic plants of the Tuscan Archipelago, Italy. Oryx 2015, 49, 1, 118-126.

3. Abdelaal, M.; Fois, M.; Fenu, G.; Bacchetta, G. Critical checklist of the endemic vascular plants of Egypt. Phytotaxa 2018, 360, 1, 19-34.

4. Jafari, F.; Zarre, S.; Gholipour, A.; Eggens, F.; Rabeler, R.; Oxelman, B. A new taxonomic backbone for the infrageneric classification of the species-rich genus Silene (Caryophyllaceae). Taxon 2020, 69, 337-368.

5. Hernández-Ledesma, P.; Berendsohn, W.G.; Borsch, T.; von Mering, S.; Akhani, H.; Arias, S.; CastañedaNoa, I.; Eggli, U.; Eriksson, R.; Flores-Olvera, H. A taxonomic backbone for the global synthesis of species diversity in the angiosperm order Caryophyllales. Willdenowia 2015, 45, 281-383.

6. Radford, E.A.; Catullo, G.; Montmollin, B.D. Important Plant Areas of the South and East Mediterranean Region: Priority Sites for Conservation; IUCN Gland (Suiza) WWF; Gland (Suiza): 2011.

7. Fakhry, A.M.; El-Keblawy, A.; Shabana, H.A.; Gamal, I.E.; Shalouf, A. Microhabitats Affect Population Size and Plant Vigor of Three Critically Endangered Endemic Plants in Southern Sinai Mountains, Egypt. Land 2019, 8, 86.

8. Hashim, A.M.; Alharbi, B.M.; Abdulmajeed, A.M.; Elkelish, A.; Hozzein, W.N.; Hassan, H.M. Oxidative stress responses of some endemic plants to high altitudes by intensifying antioxidants and secondary metabolites content. Plants 2020, 9, 869.

9. Zahran, M.; Wafaa, A.; Samy, A.; Omran, G. Endemic species in Sinai peninsula, Egypt, with particular reference to Saint Katherine protectorate: I-ecological features. J. Environ. Sci. 2015, 44, 589-609.

10. Chowdhuri, P. Studies in the genus Silene. Notes from the RGB. Edinb. 1957, 22, 221-278. 
11. Oxelman, B.; Rautenberg, A.; Thollesson, M.; Larsson, A.; Frajman, B.; Eggens, F.; Petri, A.; Aydin, Z.; Töpel, M.; Brandtberg-Falkman, A. Sileneae taxonomy and systematics. Cited 2013, 4, 2014.

12. Hosny, A.; el Hadidi, M.; Shamso, E. Taxonomic Studies of Silenoideae (Cartophyllaceae) in Egypt. 1. Systematic revision of the genus Silene L. Taeckholmia 1992, 14, 1-36.

13. Zarrinkamar, F. Foliar anatomy of the Caryophyllaceae family in Arasbaran, NW. J. Iran. Iran. J. Bot. 2001, 9, 93-102.

14. Ahmad, K.; Khan, M.A.; Ahmad, M.; Zafar, M.; Arshad, M.; Ahmad, F. Taxonomic diversity of stomata in dicot flora of a district tank (NWFP) in Pakistan. Afr. J. Biotechnol. 2009, 8, 1052-1055.

15. Keshavarzi, M.; Bozchaloyi, S.E. Leaf and stem comparative anatomical analysis of three genera of Alsinoideae (Caryophyllaceae). Iran. J. Bot. 2014, 20, 71-79.

16. Nejati, E.M.; Ghahremaninejad, F.; Attar, F. Foliar anatomy of the genus Silene L.(Caryophyllaceae) at sectional level in Iran. Iran. J. Bot 2016, 22, 2.

17. Rolland, J.; Cadotte, M.W.; Davies, J.; Devictor, V.; Lavergne, S.; Mouquet, N.; Pavoine, S.; Rodrigues, A.; Thuiller, W.; Turcati, L. Using Phylogenies in Conservation: New Perspectives; The Royal Society: 2012.

18. Rodrigues, A.S.; Gaston, K.J. Maximising phylogenetic diversity in the selection of networks of conservation areas. Biol. Conserv. 2002, 105, 103-111.

19. Hoveka, L.N.; van der Bank, M.; Bezeng, B.S.; Davies, T.J. Identifying biodiversity knowledge gaps for conserving South Africa's endemic flora. Biodivers. Conserv. 2020, 29, $2803-2819$.

20. Rasband, W.S.J.h.i.n.g.i. US National Institutes of Health: Bethesda, Maryland, USA, 2011.

21. Barthlott, W.; Neinhuis, C.; Cutler, D.; Ditsch, F.; Meusel, I.; Theisen, I.; Wilhelmi, H. Classification and terminology of plant epicuticular waxes. Bot. J. Linn. Soc. 1998, 126, 237.260.

22. R Development Core Team. R: A Language and Environment for Statistical Computing; R Foundation for Statistical Computing: Vienna, Austria, 2011.

23. Wickham, H. ggplot2: Elegant Graphics for Data Analysis; Springer: 2016.

24. Kassambara, A. ggpubr:"ggplot2" Based Publication Ready Plots (Version R Package Version 0.3. 0) [Computer software]; 2020.

25. Soetewey, A. Correlation Coefficient and Correlation Test in R. 2020. Available online: https://www.statsandr.com/blog/correlation-coefficient-and-correlation-test-in-r/.

26. Doyle, J. DNA Protocols for Plants. In Molecular Techniques in Taxonomy; Springer: 1991; pp. $283-293$.

27. Posada, D. ; K.A. Crandall. Modeltest: Testing the model of DNA substitution. Bioinformatics 1998, 14, 817818.

28. Mao, Y.R.; Zhang, Y.H.; Nakamura, K.; Guan, B.C.; Qiu, Y.X. Developing DNA barcodes for species identification in Podophylloideae (Berberidaceae). J. Syst. Evol. 2014, 52, 487-499.

29. Stace, C.A. Cuticular Studies as an Aid to Plant Taxonomy; Bulletin of the British Museum (Natural History); British Museum (Natural History): London, UK, 1965.

30. Parkhurst, D.F. The adaptive significance of stomatal occurrence on one or both surfaces of leaves. J. Ecol. 1978, 367-383.

31. Rossatto, D.R.; Kolb, R.M. Gochnatia polymorpha (Less.) Cabrera (Asteraceae) changes in leaf structure due to differences in light and edaphic conditions. Acta Bot. Bras. 2010, 24, 605-612.

32. Ullah, F.; Zafar, M.; Ahmad, M.; Shah, S.N.; Razzaq, A.; Sohail, A.; Zaman, W.; Çelik, A.; Ayaz, A.; Sultana, S. A systematic approach to the investigation of foliar epidermal anatomy of subfamily Caryophylloideae (Caryophyllaceae). Flora 2018, 246, 61-70.

33. Rohrbach, P. Monographie der Gattung Silene; W. Engelmann: 1868.

Publisher's Note: MDPI stays neutral with regard to jurisdictional claims in published maps and institutional affiliations.

(C) 2020 by the authors. Submitted for possible open access publication under the terms and conditions of the Creative Commons Attribution (CC BY) license (http://creativecommons.org/licenses/by/4.0/). 